\title{
El tiempo profundo de la infraestructura mediática'
}

\section{Deep Time of Media Infrastructure O tempo profundo da infraestrutura midiática}

DOI: https://doi.org/10.18861/ic.2019.14.2.2927

\section{SHANNON MATTERN}

matterns@newschool.edu - The New School for Social Research, Estados Unidos.

Fecha de recepción: 25 de agosto de 2019

Fecha de aceptación: 29 de septiembre de 2019

\section{RESUMEN}

En este capítulo del libro Signal Traffic: Critical Studies of Media Infrastructures, repienso la historia urbana como lahistoria delasinfraestructurasdecomunicación. Mezclando la arqueología mediática yla arqueología propiamente dicha, considero a las ciudades como lugares de mediación sónica, a través de las ondas radiales y sonoras, y como sustratos para la inscripción. Asimismo, se esbozan enfoques historiográficos y metodológicos que pueden ayudarnos a pensar en las ciudades mediáticas, la historia urbana y la historia de las infraestructuras en su conjunto.

PALABRASCLAVE: infraestructura mediática, arqueología, historia urbana.

\begin{abstract}
In this chapter of the book Signal Traffic: Critical Studies of Media Infrastructures, Irethinkurbanhistoryas a history of communication infrastructures. Blending media archaeology and archaeology proper, I consider cities as sites of sonic mediation, via radio and sound waves, as substrates for inscription. And I outline historiographic and methodological approaches that can help us to think about mediacities, urban history, and infrastructural historyin tandem.
\end{abstract}

KEYWORDS: mediainfrastructure, archaeology, urbanhistory.

\section{RESUMO}

Neste capítulo do livro Signal Traffic: Critical Studies of Media Infrastructures, repenso a história urbana como a história das infraestruturas de comunicação. Misturando arqueologia midiática e arqueologia propriamente dita, considero as cidades como locais de mediação sônica, por meio de ondas radiais e sonoras, e como substratos para a inscrição; e também esboço abordagens historiográficas e metodológicas que nos podem ajudar a pensar nas cidades-midiáticas, na história urbana e na história da infraestrutura como um todo.

PALAVRAS CHAVE: infraestrutura midiática, arqueologia, história urbana.

1 El artículo aquí traducido fue publicado en el libro Signal Traffic: Critical Studies of Media Infrastructures, editado por Lisa Parks y Nicole Starosielski (2015, University of Illinois Press). Copyright 2015 by the Board of Trustees of the University of Illinois. Usado con permiso de University of Illinois Press. Los editores de InMediaciones de la Comunicación y el traductor, Dr. Nicolás Llano Linares, agradecen enormemente a la autora, Shannon Mattern, por su generosidad, confianza y calidez durante todo el proceso. 


\section{INTRODUCCIÓN}

Cuando apareció usada por primera vez en inglés, a mediados de 1920, infraestructura se refería a carreteras, túneles, otras obras públicas y estructuras militares permanentes. El Ngram Viewer de Google, que muestra la frecuencia con que aparecen las palabras en el corpus de libros de Google, muestra que el término era más bien oscuro alrededor de 1960 -más o menos al mismo tiempo en que el término medios de comunicación comenzó a despegar-y que el uso de telecomunicaciones se generalizó. Por lo tanto, no es una coincidencia que infraestructura-una palabra de raíz latina y que denota cualquier forma de subestructura, se prestara a un uso liberal- sea comúnmente asociada con las modernas comunicaciones electrónicas y con el tráfico de señales audiovisuales.

Sin embargo, esas señales traficadas preceden largamente la era de las telecomunicaciones. Y la propia infraestructura tiene una historia mucho más extensa: ha existido tanto tiempo como la civilización. De hecho, podríamos decir quelas infraestructuras hicieron posibleslos asentimientos humanos. No solo hablo de carreteras y acueductos y desagües, el tipo de infraestructura que los arqueólogos ehistoriadores dela antigüedad examinan regularmente. También las infraestructuras mediáticas se han integrado a nuestras ciudades, sea por diseño o accidente, desde los días de Eridú y Uruk. El antropólogo Clifford Geertz (1980), el historiador urbano Peter Hall (1998), y el arqueólogo Paul Wheatley (1971) han sugerido que el nacimiento de las ciudades está enraizado tanto en la necesidad de ceremonia y comunicación como en su economía, que es la teoría dominante. Por consiguiente, las primeras ciudades tenían que ofrecer espacios propicios para la "pompa" yla comunicación. Lewis Mumford ([1938] 1966), autor de dos grandes historias de la urbanidad, concuerda en que "lo que transformó los regímenes agrícolas pasivos de la aldea en las instituciones activas de la ciudad"2 no fue simplemente un crecimiento del tamaño o densidad poblacional o económica, sino una extensión “delárea de relaciones sociales, que engendra la necesidad de combinar y cooperar, de comunicación y comunión” (p. 6). El "área de relaciones sociales” es una infraestructura: la estructura que soporta la comunicación y la comunión.

Al repensar qué constituye una infraestructura mediática, y al reconocer su profunda historia, se espera ofrecer un contrapunto útil a otro tipo de estudios sobre la temática. Quiero pensar más allá de las telecomunicaciones, más allá del siglo XIX, hacia atrás de los sistemas tecnológicos administrados por los estados modernos, las agencias gubernamentales y las corporaciones multinacionales. Tomando inspiración de los campos de la geología y el trabajo de Siegfried Zielinski (2006), nosotros - estudiosos de los medios y la infraestructura, historiadores del urbanismo, hasta ingenieros y diseñadores urbanos-

2 Nota del Traductor. Todas las citaciones que aparecen en el texto original fueron traducidas en versión libre. 
haríamos bien en examinar el tiempo profundo de la infraestructura mediática ${ }^{3}$. Y en esta forma de pensamiento más expansivo, quisiera que los que hacemos parte de los estudios de medios y de diseño consideremos qué podríamos aprender de los campos de estudio y práctica que durante mucho tiempo han examinado la infraestructura, pero que han tenido poco contacto con nuestro campo.

La arqueología y la historia urbana y arquitectónica en particular tienen mucho que ofrecer para el estudio del tráfico de señales. Por supuesto que los estudios de los medios han sido testigos de la llegada de un subcampo llamado "arqueología mediática”, en el que participan figuras como Zielinski, Friedrich Kittler, Erkki Huhtamo y Jussi Parikka. Aunque este trabajo ofrece una forma alternativa, no lineal y materialista de escribir las historias de los medios, considera a la arqueología de forma metafórica o en sentido metodológico en lugar de asumirla de manera literal. En vez de eso, quiero considerar los conocimientos delos arqueólogos de llana empuñada.

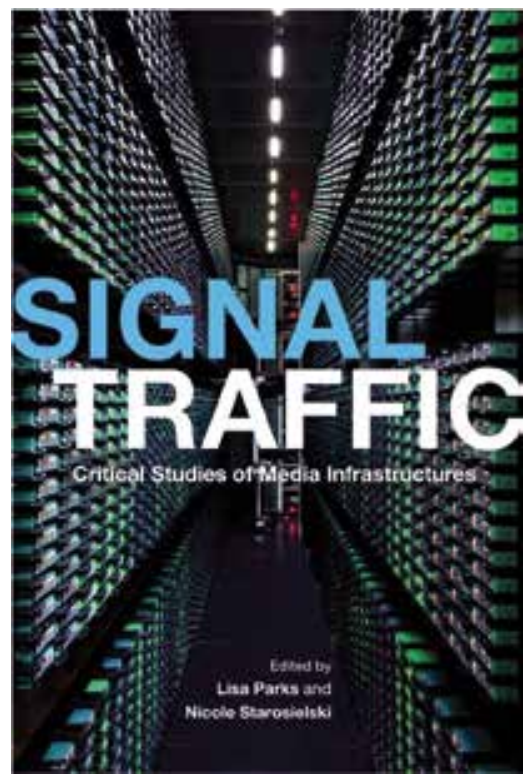

Parks, L. \& Starosielski, N. (Eds.) (2015). Signal Traffic: Critical Studies of Media Infrastructures. Illinois, U.S.: University of Illinois Press.

El historiador de la infraestructura, Paul Edwards (2003), admite que hoy en día la infraestructura "se ha convertido en un término escurridizo, a menudo utilizado para significar, esencialmente, cualquier recurso importante usado ampliamente y construido por humanos" (pp. 186 y 187); esto podría incluir el hardware, las organizaciones, "el conocimiento de fondo comunicado socialmente" -cualquier sistema sociotécnico que ofrezca una "accesibilidad casi ubicua" (p. 188). A pesar de, o tal vez debido a la flexibilidad del término, creo que quienes hacemos parte de los estudios de medios y diseño tenemos mucho que aprender de la forma en que Edwards y otros historiadores y teóricos de la infraestructura conciben y trabajan con su objeto de estudio. En la próxima sección examino lo que los arqueólogos e historiadores urbanos y arquitectónicos

3 En la edición de 2007 del taller financiado por la National Science Foundation (NSF) sobre ciberinfraestructura Paul Edwards y varios colegas abogaron por la importancia de estudiar el "largo ahora" de la ciberinfraestructura: los doscientos años de cambios políticos, culturales y técnicos de "paso lento" que han ocurrido en los últimos años "en un segundo plano" - cambios como el auge de las disciplinas científicas y la estadística- que han sentado las bases de las redes digitales (Edwards, P., Jackson, S., Bowker, G. \& Knobel, C.; enero, 2007). Por supuesto, yo defendería que los estudios sobre los medios de comunicación podrían beneficiarse de una perspectiva mucho más amplia, que reconozca que la infraestructura precede lo ciber y lo electrónico; pero, aun así, el enfoque de estos estudiosos en la contextualización histórica es útil. Y el concepto del largo ahora, un contemporáneo que se extiendehasta el pasado, complementa elllamado de Zielinski(2006) a encontrar algo nuevo en loviejo-encontrar el "ahora" en la historia- y esfuerzos recientes para considerar la "geología" de los medios de comunicación: los recursos naturales usados para hacer nuestro hardware de medios (véase: Parikka, 2013). Estos últimos esfuerzos son particularmente relevantes para nuestros esfuerzos de indagar en la historia profunda, dado que el propio concepto de tiempo profundo surgió en la geología. 
pueden decirnos acerca de cómo las ciudades antiguas ofrecían infraestructuras para la vocalidad -para discursos públicos y conversaciones- y para la escritura. Y en la sección final exploro la forma en que los métodos o unidades conceptuales de estos campos resuenan con los enfoques historiográficos de los estudios de medios y pueden incentivarnos a reflexionar críticamente sobre cómo construimos nuestras historias mediáticas, así como nuestras historias urbanas y arquitectónicas.

La meta es demostrar que pensar en términos de infraestructura puede enriquecer las investigaciones existentes dentro de los estudios de medios -en particular, los trabajos sobre la ciudad mediática- y de qué manera pensar en términos del entorno urbano puede extender nuestra visión histórica de la infraestructura mediáticay permitirnos entender más ampliamentelo que constituye una infraestructura mediática. ¿Quépodemos ganar al mirar hacia atrásen el tiempo profundo dela infraestructura mediática y su papel en la generación o en la forma de moldar nuestras ciudades? En primer lugar, desde la perspectiva de los investigadores de los medios de comunicación, podemos apreciar a los medios como potencialmente corporeizados en una escala macro, como una fuerza cuyos modos e ideologías y estéticas de operación pueden ser espacializados, y materializados, en el horizonte. Podemos leer el registro arqueológico, realizar análisisforenseso-cuando estamoslidiando con un medio comola voz, para el cual no hay artefactos coleccionables- podemos utilizar técnicas de la arqueoacústica para "escuchar" el pasado de los espacios. Podemos desenterrar los cables, sacar los alambres, trazar la epigrafía en las fachadas de los edificios, analizar los discos, y luego observar su estratificación e interconexión.

Asimismo, cuando examinamos los medios a una escala macro, también tenemos que reconocer que la historia de los medios está entrelazada con la de nuestras ciudades, sus calles y edificios, sus redes políticas, económicas y sociales, y así sucesivamente. En el proceso nos damos cuenta de que esas ciudades llevan en sí el "residuo" de todas las tecnologías mediáticas pasadas; y que, además, estos medios "pasados" no son meros artefactos o ruinas. Al igual que la categoría de lo "residual" de Raymond Williams (1977), están "formados en el pasado, pero siguen activos en el proceso cultural, no solo, y frecuentemente en absoluto, como un elemento del pasado, sino como un elemento efectivo del presente" (p. 122). Por eso nuestras ciudades actuales no son únicamente virtuales, sino simultáneamente auditivas, gráficas, textuales, sónicas, visuales y digitales. En los estudios sobre los medios de comunicación tendemos a escribir historias específicas para cada formato y a sugerir que las nuevas tecnologías sustituyen a las antiguas; pero cuando miramos nuestras historias sobre los medios de comunicación a través de nuestras ciudades, observamos una estratificación, o reverberaciones, de las épocas mediáticas. Estas realizaciones abren nuevas oportunidades metodológicas para el estudio de los medios de comunicación. 
En segundo lugar, los estudios sobre la infraestructura también tienen el potencial de contribuir a la historia urbana y arquitectónica. Por ejemplo, es posible revaluar teorías sobre el nacimiento de las ciudades, que tienden a privilegiar las explicaciones económicas acerca de la urbanización, y reforzar el papel central desempeñado por los medios y la comunicación en la historia urbana. Adicionalmente, podemos destacar el papel de la comunicación en darle forma a nuestras ciudades. Las teorías predominantes sugieren que la forma urbana está moldeada principalmente por la topografía, el transporte, la defensa, incluso por perspectivas cosmológicas o filosóficas. Sin embargo, varias formas de comunicación -ya sea la voz o las tecnologías impresas o digitales- también han moldeado a las ciudades a lo largo de la historia.

\section{TIEMPO PROFUNDO DE LA CIUDAD MEDIÁTICA}

Existe un área de estudio bien establecida, pero en constante crecimiento dentro de los estudios mediáticos, que parece prestarse al estudio interdisciplinario que estoy proponiendo aquí. Los estudiosos enfocados en la ciudad mediática han aprovechado los conocimientos de la historia y la teoría arquitectónica y urbana para pensar a los medios de comunicación en relación con lo urbano; sin embargo, han tendido a enfocar su atención en los medios modernos: fotografía, cine, televisión y otros medios similares. Existe abundancia de investigaciones sobrela arquitecturaylas ciudades en relación con imágenes fijas y en movimiento reproducidas mecánicamente. Por ejemplo, muchos historiadores de la fotografía, la arquitectura y los fenómenos culturales, inspirados en gran medida por Walter Benjamin (1969), han examinado la ciudad como un objeto fotográfico, el papel inicial de la fotografía en la documentación de la transformación urbana y como un instigador del cambio social, así como también han observado la influencia de la fotografía en determinados diseñadores urbanísticos y arquitectónicos modernos (Ackerman, 2002; Rice, 1999; Levine, 1998; Colomina, 1994; Hales, 1983; Pare, 1982). También hay una gran cantidad de trabajos sobre la ciudad y el cine como desarrollos coetáneos, la representación de la ciudad en los filmes (esta es la corriente dominante, de lejos), y la influencia de los filmes en los arquitectos y planificadores, incluyendo algunas investigaciones sobre la ciudad como una infraestructura física y social para el auge del cine (McQuire, 2008; Dimendberg, 2004; Clarke, 1997; Colomina, 1994; Eisenstein, [1938] 1989). En décadas recientes, investigadoras como Lynn Spigel (1992) y Anna McCarthy (2001) han comenzado a abordar el aumento sincrónico de la televisión y los suburbios en la postguerra, las políticas de las pantallas en los espacios públicos, y el impacto de los medios digitales en red en el diseño yla experiencia urbana (McQuire, 2009; Heckman, 2008). También ha habido excelentes trabajos sobre el impacto de la radio y las tecnologías modernas de sonido en la arquitectura, la zonificación y la expe- 
riencia urbana (Yusaf, 2014; Goodman, 2010; Bijsterveld, 2008; Larkin, 2008; Thompson, 2004).

Algunas investigaciones sobre ciudades mediáticas presentan la suposición de quela mediación de la ciudad comenzó con los medios modernos. Scott McQuire (2009), en The Media City, observa quela mediación dela experiencia urbana "ha estado en marcha al menos desde el desarrollo de las imágenes tecnológicas en el contexto de la 'modernización' urbana, a mediados del siglo XIX” (p. vii). Eric Gordon (2010), en The Urban Spectator, sitúa el origen de la ciudad mediática más tarde que McQuire: "desde la cámara de mano al final del siglo XIX hasta el teléfono móvil a finales del XX, la ciudad siempre ha sido un constructo mediado" (Gordon, 2010, p. 2). Por mi parte, sostengo que ese inicio tiene lugar mucho antes que los años finales del Siglo XIX y la era de las telecomunicaciones.

Las ciudades han sido, por supuesto, representadas durante milenios en mapas, pinturas, grabados, poemas líricos, y otros formatos mediáticos. Sin embargo, la ciudad, como "constructo mediado" ciertamente abarca mucho más que las meras representaciones de la ciudad: las tecnologías mediáticas -particularmente las infraestructuras mediáticas- se han arraigado a la evolución morfológica de nuestras ciudades desde su formación. Aunque las investigaciones sobre las ciudades mediáticas raramente han observado a la infraestructura.

Esto ha cambiado un poco durante las últimas dos décadas, con el auge de los medios digitales y locativos y de la computación ubicua, que ha inspirado a los estudiosos, diseñadores, ingenieros y artistas a prestarle mayor atención a las redes técnicas que hacen posibles nuevas formas de mediación urbana. Pero estos investigadores y profesionales raramente miran hacia atrás para observar las redes técnicas que siempre han estado allí, haciendo delas ciudades espacios comunicativos. Existe una tendencia a pasar por alto las infraestructuras que preceden a lo ciber y a lo electrónico, así como los sistemas que emergieron incluso antes del proprio término infraestructura.

En el siglo XV, por ejemplo, como ha explicado el historiador de la arquitectura Mario Carpo (2001), las nuevas tecnologías de impresión trajeron consigo nuevas infraestructuras editoriales y educativas que influenciaron dramáticamente a las prácticas de diseño (Wilson, 2005; San Juan, 2001; Favro, 1999). Los centros editoriales, con sus infraestructuras políticas-económicas, sociales y tecnológicas arraigadas, surgieron en ciudades a lo largo de Asia y Europa. El surgimiento de nuevas formas de impresión también influenció la forma en que las personas navegaban y comprendían sus ciudades. Aún hoy, las metáforas del libro informan la manera como "escribimos y leemos" nuestras ciudades: los planificadores hablando de "urbanismo legible" y de leer la ciudad como un "texto", mientras quelos diseñadores construyen aplicaciones de realidad aumentada, que superponen texto e imágenes encima de las vistas 
de la ciudad, haciendo posible una "lectura" urbana palimpsestica (Mattern, 2013a; 2014).

La voz también ha sido incorporada, desde hace mucho tiempo, en las formas urbanas. Desde sus inicios, las ciudades han sido lugares de alocución pública y conversación, y las consideraciones acústicas han informado, hasta cierto punto, el diseño y la construcción. Sin embargo, si miramos hacia atrás al ágora de Atenas o al Foro de Roma, no encontraremos infraestructuras en la forma de cableado eléctrico ni sistemas de alocución pública y escenarios con paneles acústicos. En cambio, como he argumentado en otro trabajo (Mattern, 2013b), las superficies, los volúmenes y los vacíos urbanos han funcionado como cajas y cámaras de resonancia para la mediación, también como medios de transmisión en sí mismos (gran parte de la discusión a seguir sobre la comunicación oral se basa en esa publicación). Particularmente, en casos como estos, los estudiosos de los medios pueden beneficiarse del trabajo de arqueólogos, excavando los contextos urbanos y pasados profundos de las infraestructuras mediáticas. Por ejemplo, la arqueología y su subcampo de aqueoacústica, junto con la historia urbana y arquitectónica, pueden enriquecer el entendimiento de las formas en que estos materiales han funcionado, ya sea por diseño o accidente (los arqueólogos e historiadores de la arquitectura disienten en la intencionalidad del diseño acústico antiguo), ya sea como infraestructuras del habla y la vocalidad.

"Nunca, en mi opinión”, escribe Quintilian (ca. 95 ce), "los fundadores de las ciudades habrían inducido a sus multitudes agitadas a formar comunidades si no las hubieran conmovido con la magia de su elocuencia" ( $\mathrm{s} / \mathrm{r}$ ). Aristóteles (1998), asimismo, prescribió una ciudad que no pudiera contener más personas que las que pudiera oír la voz de un heraldo, y el arquitecto Vitruvius (1914) nos dice en Siglo I a.C. sobre sus colegas diseñadores que trataron de cultivar acústicas que maximizaran la "claridad y la dulzura" (Vitruvius, 1914, p. 139) de las voces de los oradores. La historiadora de la arquitectura Diane Favro y el clasicista Christopher Johanson (2010) están creando modelos digitales del Foro Romano para entender cómo el espacio acomodaba las procesiones funerarias y, en parte, cómo funcionaba acústicamente como un espacio para el habla. Encontramos preocupaciones acústicas similares incluso antes, en la antigua Grecia. El clasicista Christopher Johnstone (2001) se ha basado en investigaciones arqueológicas para explorar cómo la arquitectura del ágora de Atenas y, más tarde, de los edificios cívicos como la estoa, los tribunales de justicia y varios auditorios, dieron forma tanto a la expresión del orador y el compromiso de su audiencia; incluso limitaron el tamaño de la audiencia, que podía ser un órgano rector o un jurado. De esta manera, dichos volúmenes urbanos afianzaron la modalidad central de la comunicación y, por lo tanto, se transformaron en medios de gobernanza y un medium principal para la socialización en la civilización antigua. 
¿Qué hay de una ciudad cuyas infraestructuras se formaron milenios después, en una era diferente de la infraestructura mediática? Consideremos a Nueva York a mediados del siglo XIX, cuando, como escribe David Henkins (1998) en City Reading, los impresos producidos masivamente cubrían la ciudad entera en la forma de carteles, signos y periódicos. Durante este periodo la imagen reproducida mecánicamente ganaba popularidad y las telecomunicaciones aumentaban. Ya en ese entonces la ciudad era un lugar de alocución pública; el medio residual delas comunicaciones orales continuaba moldeando la morfología urbana. Samuel Ruggles, uno de los constructores del Union Square de Nueva York, en 1864 afirmó que la plaza estaba "deliberadamente diseñada para apoyar la democracia participativa”.

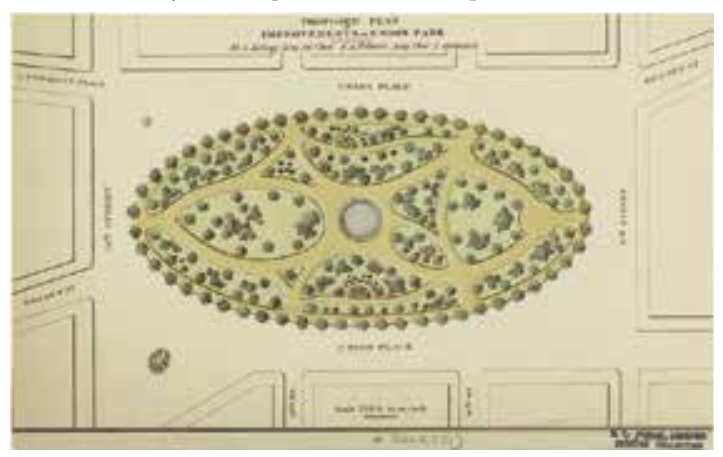

Fuente. Mid-Manhattan Library Picture Collection, New York Public Library.

Las parcelas triangulares de tierra dejadas por la imposición del parque elíptico en la cuadrícula "fueron hechas expresamente para la 'concentración degrandes masas de nuestros ciudadanos en encuentros públicos"' (MerwoodSalisbury, 2009, p. 543). A través de su continua renovación, los planificadores buscaron usar la plaza como una infraestructura para crear "ciudadanos activos e informados, así como para fomentar la armonía social” (p. 551), pero continuó y continúa siendo un lugar de reuniones y mítines radicales. Hoy en día, Union Square, como muchas otras plazas y plazuelas en Atenas, Roma y en otras ciudades antiguas del mundo, sirve como una infraestructura urbana para la integración de una variedad de medios: tecnologías locativas, mensajes de texto, pancartas de telay, aún, la vozhumana desnuda o expresada por medio de la megafonía.

Las infraestructuras de la escritura también han informado desde hace mucho tiempo cómo se formaron las ciudades. Por supuesto, las primeras superficies de escritura, hechas de arcilla y piedra, fueron los mismos materiales utilizados para construir edificios antiguos. Y a menudo esas fachadas de edifi- 
cios eran los substratos para los textos escritos. El “hábito epigráfico" distinguía a la antigua Grecia y la antigua Roma. Según Johanson, "los romanos parecían inscribir en todo" (Entrevista a Johanson, 26 de febrero, 2013). Alrededor del Foro, un antiguo podía encontrar "la palabra escrita que cubría todas las superficies de los principales monumentos" (ibíd.). Estos monumentos y fachadas de edificios no fueron diseñados para ser utilizados como substratos para la escritura -como una infraestructura arquitectónica para la comunicación-, sino que, a través de las prácticas sociales de los romanos, el "tejido dela ciudad" sirvió en última instancia para registrar las principales leyes, logros, transacciones legales y otras misivas. La ciudad estaba "archivando informalmente en su propia piel" (ibíd.).

La arqueóloga Louise Revell (2009) reconoce que estos epígrafes constituyen un "complemento natural" de la arquitectura pública en la que fueron colocados o inscritos; los escritos desempeñaron una parte integral de los procesos políticos y de los servicios religiosos; por lo cual estaban estrechamente relacionados con la práctica social de lo que era ser romano. Es importante señalar que esta mediación de la identidad romana no se adhirió a una única modalidad. El Foro proporcionó una infraestructura para la performance pública o la presentación de múltiples modos de comunicación: alocución pública, inscripción, escultura y otras formas de pompa o ceremoniales multimedia. Lo mismo puede decirse de los antiguos foros adaptados para el uso contemporáneo, aunque la mezcla de medios de hoy en día incluye tecnologías digitales entre las analógicas.

Elmundoárabeha sido igualmente rico en epigrafía. La historiadora de arte Irene Bierman (1998) describe cómo, del siglo X al XII, los fatimíes del Cairo exhibieron la escritura oficial en los exteriores de los minaretes y otras estructuras públicas. Así, al igual que en Grecia y en Roma, la arquitectura funcionaba como una infraestructura para comunicar reclamos territoriales y codificar creencias. Como sostiene Bierman, las propiedades estéticas específicas de esos "textos públicos" (p. 20) -su color, materialidad y forma- desempeñaron un papel clave en cómo y qué comunicaban. El historiador de arte Robert Harrist (2008) hace afirmaciones similares sobre la Moya china, escritos en piedra que funcionaron como "paisaje" y que, "a través de su localización y su interacción con el mundo natural, incorporan memoria histórica en la topografía de China yevocan mundos míticos que trascienden las experiencias dela vida cotidiana" (p. 23).

Pero la escritura no está inscrita simplemente en las paredes de nuestras ciudades. Mumford ([1938] 1966) y Harold Innis (1951) discuten la escritura como algo central del auge del comercio, la contabilidad y la gobernanza y, por lo tanto, de la administración de las primeras ciudades. La escritura es una infraestructura urbana-política-económica integral. El antropólogo Brinkley Messick (1993) argumenta que incluso podemos encontrar paralelismos entre 
la escritura y la forma urbana centrando el examen en la historia de las inscripciones arquitectónicas islámicas y sus paralelismos formales en la propia articulación del espacio urbano. Messick discute los llamados "textos en espiral" arábigos, textos en los que la escritura gira en forma de espiral, entrelazando forma y contenido, y argumenta que "esta poética del espacio escrito puede, entonces, extenderse a los dominios generales de la organización espacial: ciudades, arquitectura y el espacio del estado" (p. 231).

Imagen 2. Forma urbana en espiral en Tảizz, Yemen. Ta’izz con Mesquita Aschrifayya.

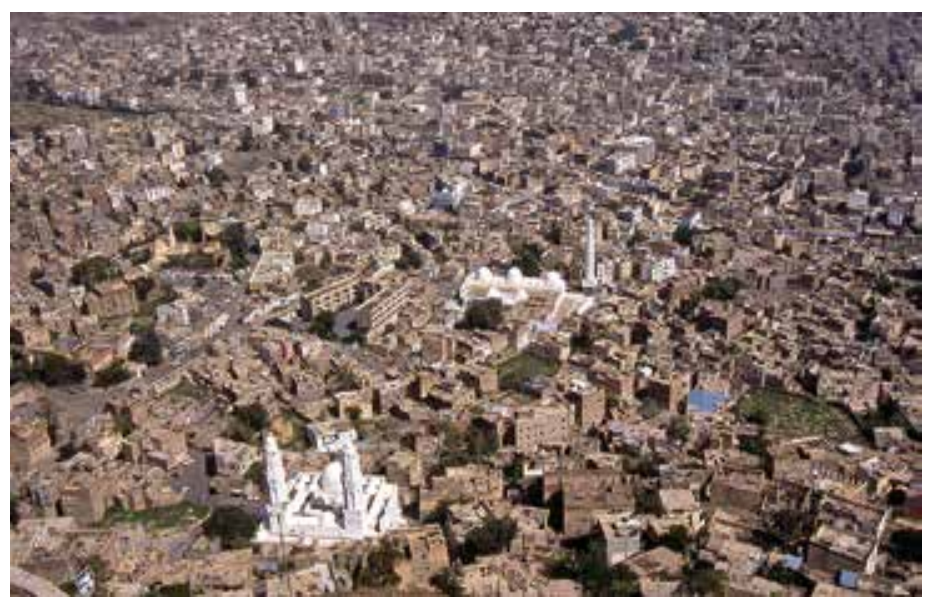

Fuente. Wikimedia Commons, CC BY-SA 3.0, http://en.wikipedia.org/wiki/File:Taizz.jpg\#file.

El antropólogo contrasta "la escritura urbana curvilínea" (p. 246) del pueblo yemení de Ibb — que describe como "un laberinto de casas de varios pisos muy próximas y repletas, en callejones estrechos y sinuosos y calles sin salida, con abundantes "espacios irregulares, residuales" (pp. 246 y 247) — con las regiones más nuevas, zonificadas y planificadas, caracterizadas por "calles relativamente rectasyamplias, con un poco deespacioentrelosedificios"(Ibíd.).Estanueva separación y precedencia de la forma urbana sobre el contenido urbano, la evolución paralela en la forma urbana, Messick argumenta, "es análoga al cambio de los textos en espiral a sus sucesores enderezados" (ibíd.). El hecho de que podamos o no argumentar una relación causal tal vez no venga al caso; lo que vemos aquí es una resonancia morfológica entre una infraestructura integral política-económica y una infraestructura-mediática cultural, y la forma de la propia ciudad.

En la actualidad, los teóricos de la arquitectura y el urbanismo parecen estar dispuestos a postular relaciones deterministas formales entre las infraestructuras digitales y nuestras nuevas "ciudades inteligentes" en Songdo, en Corea, o en Masdar, en los Estados Unidos. Los constructores de estos desarrollos en red frecuentemente eliminan o minimizan el diseño de oportunidades para 
modos de comunicación no planificados (y no modernos): las calles parecen destinadas principalmente a trasladar personas de una estación de telecomunicaciones a otra, en lugar de fomentar las interacciones cara a cara; y las fachadas de los edificios se construyen con materiales anti-grafiti.

Parece que, en tales lugares, hay poca infraestructura de medios "residual" para excavar. Sin embargo, ya ha surgido un enorme contingente de críticos que argumentan que tales desarrollos, al contradecir milenios de experiencia en diseño urbano, están destinados a fracasar ${ }^{4}$. Como ha argumentado Richard Sennett (4 de diciembre, 2012), estas ciudades sobre-zonificadas, sobreracionalizadas y desprovistas de toda sensibilidad histórica desafían el hecho de que el desarrollo real en las ciudades es a menudo azaroso, o está entre las grietas de lo permitido. De acuerdo con Sennett, "el peligro ahora es que esta ciudad rica en información tal vez no haga nada para ayudar a las personas a pensar por sí mismas o comunicarse bien con los otros" (ibíd.). Una ciudad mediática que no prevé una superposición de infraestructuras comunicativas, que borra el tiempo profundo de la mediación urbana, es más pasmosa que inteligente, más máquina que metrópoli.

Estos ejemplos demuestran que nuestras historias mediáticas están profundamente conectadas con nuestras historias urbanas y arquitectónicas (yconlos futuros); y que, en muchos casos, estas formas culturales y tecnológicas se construyen mutuamente. Por lo tanto, en el estudio específico del tiempo profundo de nuestras infraestructuras mediáticas, los investigadores y los profesionales de todos los campos deben considerar estos sistemas en relación unos con otros. Es más, tenemos que reconocer que la integración de estas diversas estructuras forma las prácticas sociales y las experiencias cotidianas de las personas que viven con ellas, y es simultáneamente formada por ellas.

\section{MÉTODOS PARA EXCAVAR EN EL TIEMPO PROFUNDO DE LAS INFRAESTRUCTURAS}

En esta sección final, examino cómo los métodos y conceptos centrales de la arqueología, la historia urbana y los estudios de la infraestructura resuenan con los enfoques metodológicos de los estudios de medios, y cómo estas herramientas intelectuales "importadas" de esas áreas pueden incentivarnos a pensar más críticamente sobre cómo construimos nuestras historias de los medios de comunicación. Espero también reforzar las sugerencias hechas en la sección anterior: pensar en términos de infraestructura puede enriquecer las investigaciones de los medios de comunicación sobre la ciudad mediática; y pensar en términos del ambiente urbano puede extender el alcance histórico de la infraestructura mediática y puede permitir una comprensión más amplia de lo que constituye una infraestructura.

4 Al respecto, pueden consultarse: Mattern (5 de noviembre, 2014); y los trabajos publicados en: London School of Economics (2012); y en City in a Box -volume 34- (diciembre, 2012). 
A continuación, esbozo seisleccioneshistoriográficaso metodológicas que surgen de pensar la ciudad mediática en relación con la infraestructura y de pensar sobre la infraestructura mediática en relación con la historia urbana. De ninguna manera estas seis lecciones, o conceptos, son mutuamente excluyentes; en realidad, hay un poco de redundancia, pero creo que, en algunos casos, reafirmar el mismo principio usando un lenguaje diferente solo puede mejorar su utilidad potencial.

\subsection{Enredos tecno-socio-espacio-materiales}

El tiempo profundo de la mediación urbana se manifiesta en el estrato material: en estratificación literal. Henri Lefebvre ([1974] 1991) ha argumentado que el espacio urbano está formado por regímenes de capitales superpuestos y las infraestructuras que crean en su propia imagen; el resultado, que Lefebvre ha sugerido de forma célebre, no es diferente de una hojaldrada masa de milhojas. Pero el palimpsesto no es una mera metáfora.

En su excelente estudio de la infraestructura en la Nigeria urbana, el antropólogo Brian Larkin (2008) escribe que "la forma física de la ciudad emerge de la estratificación de infraestructuras (...), a lo largo del tiempo” (p. 5). La naturaleza de esa estratificación, sin embargo, no es una mera suplantación u obsolescencia. Si excavamos a través de los estratos, encontramos mucho más que ruinas (y aquí es donde, creo, la metáfora arqueológica a veces puede ser un poco engañosa). Excavando estas capas, a menudo encontramos que, dependiendo de diferentes factores contextuales, varias infraestructuras tienen temporalidades y trayectorias evolutivas distintivas.

Como he argumentado en otra parte (Mattern, 2013b), a través de la "excavación" podemos evaluar la vida útil de varias infraestructuras mediáticas y determinar cuándo las "viejas" infraestructuras "se filtran" en los paisajes de los nuevos medios de comunicación, cuándo los medios de diferentes épocas están estratificados de forma palimpsestica, o cuándo las nuevas infraestructuras "remedian" a sus predecesores. Los geógrafos Stephen Graham y Simon Marvin (1996) expresan que:

debido a los costos de desarrollar nuevas redes de telecomunicaciones, [por ejemplo], se hace todo lo posible por enlazar las fibras ópticas a través de conductos de agua, gas y aguas residuales; entre las ciudades, a menudo se utilizan las rutas ferroviarias, carreteras y vías fluviales existentes. (p. 329; cursiva del traductor).

Y en el Foro Romano, como explicó Johanson (Entrevista a Johanson, 26 de febrero, 2013), la escultura, la arquitectura, la epigrafía yla alocución pública se reforzaron mutuamente en el espectáculo de la procesión fúnebre y otras pompas públicas. La misma arquitectura que sirvió como caja de resonancia para 
la alocución pública también sirvió como sustrato para la epigrafía; y hoy sirve como sustrato para el grafiti y como andamiaje para las antenas de los teléfonos celulares. Las infraestructuras históricas de los medios de comunicación en los "niveles inferiores" de nuestras ciudades están a menudo muy vivas y siguen dándole forma a la ciudad contemporánea. Son los "residuos" de Williams. Esta mezcla de temporalidades se ajusta a la definición de Christopher Witmore (2006) del tiempo arqueológico: "el enredo, el entremezclado, el quiasma de pasados y presentes" (p. 279).

\subsection{Historias en red}

Graham y Marvin (2001) enumeran algunas de esas capas infraestructurales entremezclas - "superpuestas, disputadas e interconectadas"-, o lo que ellos llaman de escapes ${ }^{5}$ : la “electropolis' de la energía y el poder" (p. 8), "la 'hidrópolis" del agua ylos residuos" (Ibíd.), "la cciberciudad' dela comunicación electrónica” (Ibíd.). Pero al tomar la visión a largo plazo sobre esta entremezcla, es posible entender estos escapes enredados el uno con el otro, no solo espacialmente sino también temporalmente. La historia de cualquiera de estos escapes está conectada e inextricablemente enlazada a las historias de los otros; de la misma manera que, como vimos en los ejemplos arqueológicos anteriores, nuestras historias dela infraestructura mediática están profundamente conectadas con nuestras historias urbanas y arquitectónicas.

Richard John (2000) sugiere que

el concepto de una infraestructura de la información [por ejemplo] (...) destaca el hecho de que la transmisión de información ha sido coordinada desde hace mucho tiempo por una constelación de instituciones, en lugar de por una sola agencia gubernamental o empresa comercial. (p. 56)

Como se mencionó anteriormente, esa constelación infraestructural incluye no solo a las instituciones, sino también las prácticas cotidianas de la gente común. Es importante reconocer la codependencia, el entrelazamiento de estas diversas entidades y sistemas: el telégrafo y el teléfono, el ferrocarril y el telégrafo, las infraestructuras de transporte y el sistema postal, las infraestructuras de impresión y escritura, la escritura y la alocución oral, la arquitectura y la inscripción, y los diversos sistemas sociales y regulatorios. Tal vez también sería importante escribir sus historias juntas.

Edwards y otros autores (Edwards et al., enero, 2007) plantean un marco general sobre cómo podrían formarse estas "constelaciones" (al menos en el mundo de la ciberinfraestructura). Comienza con la construcción de sistemas; luego la transferencia de tecnología entre dominios; la aparición de variaciones en el diseño original del sistema y la aparición de sistemas competitivos;

5 Nota del Traductor. El término original en ingles es scapes. 
la eventual fusión de estos diversos sistemas a través de pasarelas, en redes; la estandarización de estas redesy su fusión en interredes -con, al tiempo que, "las opciones tempranas limitan las opciones disponibles más adelante” (pp. Iy II). Tal modelo podría parecer bastante determinista para aquellos que miramos a la tecnología desde una orientación humanística, o para aquellos que somos constructivistas. Sin embargo, creo que este modelo identifica varias fases o puntos centrales, que ocurren durante la maduración de los sistemas tecnológicos que ya reconocemos y que deberíamos animarnos a buscar. Como se sugiere en Edwards et al., "modelar" la formación de estas "constelaciones" infraestructurales en red no implica que sean sistemas rígidamente entrelazados:

\begin{abstract}
El eventual crecimiento de una infraestructura compleja y las formas que toma son el resultado de historias convergentes, dependencias del camino, serendipia, innovación y "bricolaje" (intentar arreglar). Hablar de ciberinfraestructura como una máquina a ser construida o un sistema técnico a ser diseñado tiende a restarle valor a la importancia de los problemas sociales, institucionales, organizacionales, legales, culturales y otros problemas no técnicos que los desarrolladores siempre enfrentan. (pp. 6 y 7 )
\end{abstract}

Estas innumerables infraestructuras necesitan estar conectadas en red a nuestras historiografías de las infraestructuras mediáticas. También es importante situar a esas historias en red historias dentro de la longue durée - para reconocer, como lo hace John (2000), que los sistemas y las instituciones han sido "coordinadas desde mucho tiempo" en una infraestructura de información; o, como indican Edwards et al. (enero, 2007), que sus constelaciones son el resultado dehistorias convergentes. Así que, en lugar de simplemente examinar los aspectos técnicos, sociales, institucionales y los sistemas culturales entrelazados que dieron origen, por ejemplo, a la ciberinfraestructura, podríamos reconocer que esta infraestructura de información en particular está conectada en red con la larga historia de las infraestructuras de la información. La información en sí misma tiene un tiempo profundo, como han revelado los estudiosos de la historia intelectual y los bibliotecarios (Day, 2001; Gleick, 2012).

\title{
3.3. Dependencias del camino
}

La dependencia del camino que referencian Edwardsyotros autores (enero, 2007), es un concepto particularmente útil para los estudiosos a los que se les ha enseñado a evitar a toda costa que se los etiquete de tecnodeterministas, que, como bromea Geoffrey Winthrop-Young (2011), "es un poco como decir que a [uno] le gusta estrangular perritos monos" (p. 121). Tal pensamiento sospechoso a menudo sale a la luz en la retórica de las "ciudades inteligentes". Allí, la ciudad, típicamente construida tabula rasa, es equiparada con su infraestructura tecnológica: la red digital es la ciudad. Sin embargo, pocos viven en ciudades que nacen de la noche a la mañana; la mayoría de las metrópolis son el producto 
de décadas, siglos, o milenios de expansión y renovación, arrasamiento y reconstrucción, relleno y estratificación. Al pensar en cómo interactúan estas capas, los estudiosos delas humanidades, en nuestra sobre-compensación para evitar el TD (Technological Determinism) escarlata, a menudo nos resistimos a reconocer la existencia de caminos bien transitados y la forma cómo han limitado las opciones futuras. Vemos estos caminos en la evolución a largo plazo de lasinfraestructuras mediáticas delas ciudades. El historiador dela arquitectura Kazys Varnelis (2005) ofrece un ejemplo concreto dela potencia delos caminos:

\begin{abstract}
Las nuevas infraestructuras no reemplazan a las antiguas, sino que se montan sobre ellas, formando palimpsestos físicos y organizativos -las líneas telefónicas siguen las líneas ferroviarias, y con el tiempo estas trayectorias no se han difuminado, sino mejor, grabado más profundamente en el paisaje urbano (pp. 27 y 28 ).
\end{abstract}

Por lo tanto, es posible rastrear esos caminos infraestructurales hasta la historia profunda. Hacerlo obliga a reconocer que aquellos espacios construidos para acomodar formas históricas de comunicación también "informan" y funcionan como parte de las infraestructuras mediáticas actuales. El modelo conceptual de la dependencia del camino equilibra el reconocimiento de que las tecnologías tienen efectos materiales -los canales y espacios tendidos por las tecnologías precedentes sí dirigen el desarrollo, hasta cierto punto, de las tecnologías sucesoras-con el reconocimiento de los roles desempeñados por la serendipia y los intentos de arreglar, por factores históricos sociales y culturales, en el desarrollo tecnológico.

\title{
3.4. Las personas como infraestructura
}

El registro material histórico muestra que las personas no han sido simples beneficiarios de infraestructuras, sino que en realidad ellos mismos han servido como infraestructuras. Si, por ejemplo, el suministro público de agua no se extiende a un barrio en particular, los residentes de ese vecindario llenarán a menudo sus tanques y baldes dentro de la zona de servicios yllevarán el agua esa última milla a casa. La gente, en otras palabras, hace el trabajo de las bombas y tuberías ausentes. Este ha sido el caso durante milenios. Hay muchos paralelos con la infraestructura mediática. Por ejemplo, como Greg Downey (2002) ha argumentado convincentemente, los mensajeros eran un enlace central en la red de telégrafos (Downey, 2014). En la antigua Roma, como explicó Johanson (Entrevista a Johanson, 26 de febrero, 2013), los residentes transformaron cada superficie del entorno construido como un sustrato para la escritura, y las personas usaban sus voces para convertir los volúmenes y las superficies de las ciudades antiguas en cámaras de resonancia para la alocución pública. Si no se distribuyeran los anuncios públicos importantes a las zonas urbanas 
periféricas, los residentes de esas áreas se transportarían hasta el centro de la ciudad para escuchar o leer las noticias.

Como sostiene AbdouMaliq Simone (septiembre, 2004), incluso hoy en África -y, sin duda, en gran parte del Sur Global y a lo largo de gran parte de la historia global- las personas frecuentemente compensan las "infraestructuras urbanas subdesarrolladas, utilizadas en exceso, fragmentadas y, a menudo, improvisadas" (p. 425). Las "incesantemente flexibles, móviles y provisionales intersecciones de residentes (...) operan sin nociones claramente delineadas de cómo la ciudad debe ser habitada y usada" (p. 407); y ellos mismos completan donde sus cables y tuberías se quedan cortas. Mirar a través de la longue durée el papel que las personas han jugado en las constelaciones infraestructurales nos ayuda a apreciar la centralidad profundamente afianzada y continua del biopoder yla labor intelectual humana en nuestras constelaciones infraestructurales (automatizadas, digitales o de otro tipo).

\subsection{Informal / desarrollo sombra}

La mención de Simone (septiembre, 2004) de lo flexible, lo móvil y lo provisional sugiere que la historia de la infraestructura -y la historia de los medios en general- ha sido profundamente informada por desarrollos informales y "sombra". En muchas partes del mundo en desarrollo (incluso en el desarrollado), donde las instituciones no proporcionan, y tal vez nunca lo han hecho, el acceso universal a servicios públicos como los medios de comunicación, las islas de acceso dentro de mares de exclusión son la norma. Esto es cuando la gente típicamente "se rebela". Larkin (2008) escribe sobre el fraude, la reutilización o la piratería de las infraestructuras existentes en Nigeria. Tales improvisaciones han aparecido a lo largo de las historias de los medios -como en la cooptación de fachadas de edificios como sustratos para la escritura pública en la antigua Roma y Egipto-y estas prácticas periféricas deberían hacer parte de nuestras historias mediáticas e infraestructurales. Consideremos la larga historia de personas haciendo marcas no oficiales, grafitis en muros urbanos; o la larga historia de publicaciones pirateadas y mercados urbanos para textos no autorizados; o la extensa historia de personas haciendo ruido no autorizado -haciendo proselitismo o anunciando sus mercancías- en el espacio público (Johns, 2010; Baird \& Taylor, 2011; Schwartz, 2011).

Pensar en el tiempo profundo de la infraestructura de los medios -más allá de aquellos sistemas tecnológicos administrados por los estados modernos, las agencias gubernamentales y las corporaciones multinacionales- revela que a medida que las infraestructuras se han ido institucionalizado, centralizando y conectando en red, también ha evolucionado lo que constituye la "informalidad". Situar a las infraestructuras informales en relación con la larga historia de la infraestructura revela el hecho de que la "sombra" de una infraestructura también tiene una historia. 


\subsection{Escala}

Al examinar las infraestructuras de la vocalidad y la escritura he considerado entidades tan pequeñas como la voz individual y tan grandes como una forma urbana completa. Las infraestructuras actuales, por supuesto, abarcan redes globales e incluso dominios extraterrestres. Así, las infraestructuras nos obligan a pensar en la granularidad de las observaciones. Graham y Marvin (1996) enumeran varias escalas de análisis infraestructural, incluyendo la corporal, local, urbana, regional, nacional, internacional y global. Al escribir historias mediáticas-infraestructurales importa si se está escribiendo historias de los objetos mediáticos, historias de los medios locales, historias de los medios urbanos, historias de los medios nacionales, o historias de los medios culturales, y escoger entre estas puede ser complicado por el hecho de que las infraestructuras se extienden a través de estas escalas, conectando tecnologías a redes e inter-redes.

Edwards (2003) sugiere que la escala no tiene que ser concebida como una mera cualidad geográfica: también es posible considerar escalas de fuerza (desde el cuerpo humano a la geofísica), escalas de tiempo (desde el tiempo humano hasta el tiempo geofísico) y escalas de organización social (de individuos a gobiernos). Una vez más, las infraestructuras abarcan todas estas escalas. Y esas escalas - lo que constituye la "nación", o cómo se conciben los límites del cuerpo "subjetivado", por ejemplo-también tienen una historia profunda.

La visión macro-espacio-temporal es particularmente esclarecedora en el sentido que obliga a considerar las formas de los medios y las infraestructuras en relación con sus funciones a largo plazo - "las razones por las que llegaron a existir en primer lugar” (Edwards, 2003, p. 204). En lugar de pensar en cómo el telégrafo suplantó al servicio postal, o cómo la escritura suplantó a la voz, por ejemplo, estos dos sistemas pueden ser pensados como dos instancias de un propósito infraestructural compartido. Como sugiere Edwards, contextualizar el teléfono, el telégrafo, el correo y otras tecnologías modernas dentro del concepto de la revolución de control de James Beniger "nos permite comprender no solo la génesis y el crecimiento de las muchas grandes infraestructuras que caracterizan a la modernidad, sino también el proceso de vincular a estas infraestructuras entre sí” (p. 207). Por supuesto, tendríamos que identificar propósitos infraestructurales alternativos para también abarcar nuestras infraestructuras premodernas. Cualquiera que sean esas temáticas o ideologías intencionadas, este acto de vincular y contextualizar pone en primer plano la continuidad histórica (y tal vez algunas discontinuidades) entre infraestructuras -el largo ahora, el "tiempo profundo"- y las innumerables estructuras que se han entrelazado para permitirnos traficar con señales de innumerables formas a lo largo de las épocas. 


\section{REFERENCIAS}

Ackerman, J. (2002). Origins, Imitations, Conventions: Representation in the Visual Arts. Cambridge: MIT Press.

Aristóteles (1998). Politics. In J. Barnes (ed.), Complete Works of Aristotle. Princeton, N.J.: Princeton University Press.

Baird, J. \& Taylor, C. (2011). Ancient Graffiti in Context. New York: Routledge.

Benjamin, W. (1969). Illuminations. New York: Schocken.

Bierman, I. (1998). Writing Signs: The Fatimid Public Text. Los Angeles: University of California Press.

Bijsterveld, K. (2008). Mechanical Sound: Technology, Culture, and Public Problems of Noise in the Twentieth Century. Cambridge: MIT Press.

Carpo, M. (2001). Architecture in the Age of Printing: Orality, Writing, Typography, and Printed Images in the History of Architectural Theory. Cambridge: MIT Press.

City in a Box (December, 2012). Archis, 34. Recuperado de: http://archis.org/ publications/volume-34-city-in-a-box/

Clarke, D. (ed.) (1997). The Cinematic City. New York: Routledge.

Colomina, B. (1994). Privacy and Publicity: Architecture as Mass Media. Cambridge: MIT Press.

Day, R. (2001). The Modern Invention of Information: Discourse, History, and Power. Carbondale: Southern Illinois University Press.

Dimendberg, E. (2004). Film Noir and the Spaces of Modernity. Cambridge: Harvard University Press.

Downey, G. (2002). Telegraph Messenger Boys: Labor, Technology, and Geography, 18501950. New York: Routledge.

Downey, G. (2014). Making Media Work:Time, Space, Identity, and Labor in the Analysis of Information and Communication Infrastructure. In Gillespie, T., Boczkowski, P. \& Foot, K. (Eds.), Media Technologies: Essays on Communication, Materiality and Society (pp. 141-165). Cambridge: MIT Press.

Edwards, P. (2003). Infrastructure and Modernity: Force, Time, and Social Organization in the History of Sociotechnical Systems. In Misa, T., Brey, P. \& Feenberg, A. (ed.), Modernity and Technology (pp. 185-225). Cambridge: MIT Press.

Edwards, P., Jackson, S., Bowker, G. \& Knobel, C. (January, 2007). Understanding Infrastructure: Dynamics, Tension, and Design. In History and Theory of Infrastructure: Lessons for New Scientific Cyberinfrastructures. Universidad de Michigan: Ann Arbor. 
Eisenstein, S. ([1938] 1989). Montage and Architecture. Assemblage, 10, 111-131.

Entrevista a C. Johanson (26 de febrero, 2013).

Favro, D. \& Johanson, C. (2010). Death in Motion: Funeral Processions in the Roman Forum. Journal of the Society of Architectural Histofebrurians, 69(1), 15

Favro, D. (1999). Meaning and Experience: Urban History from Antiquity to the Early Modern Period. Journal of the Society of Architectural Historians, 58(3), 364-373

Geertz, C. (1980). Negara: The Theatre State in Nineteenth-Century Bali. Princeton, N.J.: Princeton University Press.

Gleick, J. (2012). The Information: A History, a Theory, a Flood. New York: Vintage.

Goodman, S. (2010). Sonic Warfare: Sound, Affect, and the Ecology of Fear. Cambridge: MIT Press.

Gordon, E. (2010). The Urban Spectator:American Concept-Citiesfrom Kodak to Google. Hanover, N.H.: Dartmouth College Press.

Graham, S. \& Marvin, S. (1996). Telecommunications and the City: Electronic Spaces, Urban Places. New York: Routledge.

Graham, S. \& Marvin, S. (2001). Splintering Urbanism: Networked Infrastructures, Technological Mobilities and the Urban Condition. New York: Routledge.

Hales, P. (1983). Silver Cities: Photographing American Urbanization, 1839-1915. Philadelphia: Temple University Press.

Hall, P. (1998). Cities in Civilization. New York: Pantheon.

HarristJr., R. (2008). The Landscape of Words: Stone Inscriptions from Earlyand Medieval China. Seattle: University of Washington Press.

Heckman, D. (2008). Small World: Smart Houses and the Dream of the Perfect Day. Durham, N.C.: Duke University Press.

Henkins, D. (1998). City Reading: Written Words and Public Spaces in Antebellum New York. New York: Columbia University Press.

Innis, H. (1951). The Bias of Communication. Toronto: University of Toronto Press.

John, R. (2000). Recasting the Information Infrastructure for the Industrial Age. In Chandler, A. \& Cortada, J. (Eds.). A Nation Transformed: How Information Has Shaped the United States from Colonial Times to the Present (pp. 74-88). New York: Oxford University Press.

Johns, A. (2010). Piracy: The Intellectual Property Warsfrom Gutenberg to Gates. Chicago: University of Chicago Press. 
Johnstone, C. (2001).Communicating in Classical Contexts: The Centrality of Delivery. Quarterly Journal of Speech, 87(2), 121-143.

Larkin, B. (2008). Signal and Noise: Media, Infrastructure, and Urban Culture in Nigeria. Durham, N.C.: Duke University Press.

Lefebvre, H. ([1974] 1991). The Production of Space. Oxford: Blackwell.

Levine, N. (December, 1998). 'The Significance of Facts': Mies's Collages Up Close and Personal. Assemblage, 37, 70-101.

London School of Economics (2012). Electric City. London: LSE. Recuperado de:http:// lsecities.net/publications/conference-newspapers/the-electric-city

Mattern, S. (2013b). Ear to the Wire: Listening to Historic Urban Infrastructures. Amodern. Recuperado de: http://amodern.net/article/ear-to-the-wire

Mattern, S. (April 28, 2014). Interfacing Urban Intelligence. Places. Recuperado de: https://placesjournal.org/article/interfacing-urban-intelligence

Mattern, S. (January 14, 2013a). Paju Bookcity: The Next Chapter. Places. Recuperado de: https://placesjournal.org/article/paju-bookcity-the-next-chapter

Mattern, S. (November 5, 2014). Methodolatry and the Art of Measure. Places. Recuperado de: https://placesjournal.org/article/methodolatry-and-the-art-ofmeasure

McCarthy, A. (2001). Ambient Television: Visual Culture and Public Space. Durham, N.C.: Duke University Press.

McEwen, I. (1993). Hadrian's Rhetoric I: The Parthenon. RES: Anthropology and Aesthetics, 24, 55-66.

McQuire, S. (2008). The Media City: Media, Architecture and Urban Space. Thousand Oaks, U.S.: Sage.

McQuire, S., Martin, M. \& Niederer, S. (eds.) (2009). Urban Screens Reader, Institute of Network Cultures Reader. Amsterdam: Institute of Network Cultures and Creative Commons.

Merwood-Salisbury, J. (2009). Patriotism and Protest: Union Square as Public Space, 1832-1932. Journal of the Society of Architectural Historians, 68(4), 540-559.

Messick, B. (1993). The Calligraphic State: Textual Domination and History in a Muslim Society. Berkeley: University of California Press.

Mumford, L.([1938] 1966). The Culture ofCities. New York: Harcourt Brace Jovanovich.

Pare, R. (1982). Photography and Architecture: 1839-1939. Montreal: Canadian Center for Architecture. 
Parikka, J. (August 20, 2013). Deep Times and Geology of Media. Machinology. Recuperado de: http://jussiparikka.net/2013/08/20/deep-times-and-geologyof-media

Quintilian (ca. 95 ce). Institutio Oratoria. Recuperado de: http://perseus.uchicago.edu/ perseus-cgi/citequery3.pl?dbname $=$ LatinAugust $2012 \&$ getid $=1 \&$ query $=$ Qui nt.\%202.16.15

Revell, L. (2009). Roman Imperialism and Local Identities. New York: Cambridge University Press.

Rice, S. (1999). Parisian Views. Cambridge: MIT Press.

San Juan, R. M. (2001). Rome: A City Out of Print. Minneapolis: University of Minnesota Press.

Schwartz, H. (2011). Making Noise: From Babel to the Big Bang and Beyond. Brooklyn: Zone.

Sennett, R. (December 4, 2012). No One Likes a City That's Too Smart. Guardian.

Simone, A-M. (September 2004). People as Infrastructure: Intersecting Fragments in Johannesburg. Public Culture, 16, 407-425.

Spigel, L. (1992). Make Room for TV: Television and the Family Ideal in Postwar America. Chicago: University of Chicago Press.

Thompson, E. (2004). The Soundscape of Modernity: Architectural Acoustics and the Culture of Listening in America, 1900-1933. Cambridge: MIT Press.

Varnelis, K. (2005). Centripetal City. Cabinet 17, 27-28.

Vitruvius (1914). The Ten Books on Architecture. Cambridge: Harvard University Press.

Wheatley, P. (1971). The Pivot of the Four Quarters: A Preliminary Enquiry into the Origins and Character of the Ancient Chinese City. Chicago: Aldine.

Williams, R. (1977). Marxism and Literature. New York: Oxford University Press.

Wilson, B. (2005). The World in Venice: Print, the City, and Early Modern Identity. Toronto: University of Toronto Press.

Winthrop-Young, G. (2011). Kittler and the Media. Malden: Polity.

Witmore, C. (2006). Vision, Media, Noise, and the Percolation of Time: Symmetrical Approaches to the Mediation of the Material World. Journal of Material Culture 11(3), 267-292.

Yusaf, S. (2014). Broadcasting Buildings: Architecture on the Wireless, 1927-1945. Cambridge: MIT Press. 
Zielinski,S. (2006).Deep Time of the Media:Towardan Archaeology of Hearingand Seeing by Technical Means. Cambridge: MIT Press.

${ }^{*}$ Contribución: el 100\% del trabajo pertenece a la autora.

\section{(c) BY}

Artículo publicado en acceso abierto bajo la Licencia Creative Commons - Attribution 4.0 International (CC BY 4.0).

\section{IDENTIFICACIÓN DE LAAUTORA}

Shannon Mattern. Doctora en el área Cultura y Comunicación por la New York University, Estados Unidos. Profesora en el Departamento de antropología en The New School for Social Research, Estados Unidos. Su producción escrita y la docencia se centran en las arquitecturas e infraestructuras de los medios de comunicación y en las epistemologías espaciales. Es autora de tres libros: The New Downtown Library: Designing with Communities (2007), Deep Mapping the Media City (2015); y Code and Clay, Dirt and Data: 5000 Years of Urban Media (2017), todos publicados por la University of Minnesota Press. Ha escrito también numerosos capítulos de libros, artículos en revistas académicas y contribuye regularmente con una columna en Places Journal. Parte de esos materiales y trabajos pueden consultarse en: wordsinspace.net.

\section{IDENTIFICACIÓN DEL TRADUCTOR}

Nicolás Llano Linares. Doctor en Ciencias de la Comunicación por la Universidade de São Paulo, Brasil. Realizó un intercambio de investigación en la University of Copenhagen, Dinamarca, en los departamentos de Cine y Ciencias Sociales (2014-2015), y formó parte del curso “Code Societies", en la School for Poetic Computation (Nueva York, 2018). Autor del libro 5-5 (Salvaje, 2016) y co-editor de Comunição \& Antropologia Visual (Inmod, 2014). Es profesor del curso "Escritura Experimental y otras estrategias de creación no autorizadas”, Escola da Cidade, São Paulo, Brasil.

\section{REGISTRO BIBLIOGRÁFICO}

Mattern, S. (julio-diciembre, 2019). El tiempo profundo de la infraestructura mediática. InMediaciones de la Comunicación, 14(2), 211-232 